EVS25

Shenzhen, China, Nov 5-9, 2010

\title{
CITYELEC: A global solution approach for the electrification of urban mobility in Spain
}

\author{
I. Iglesias, J.J. Valera \\ Transport unit, Tecnalia Research \& Innovation, Parque Tecnológico Zamudio 202 \\ E-48170 Vizcaya, Spain \\ inaki.iglesias, juanjose.valera@tecnalia.com
}

\begin{abstract}
Urban mobility electrification is a high complex optimization problem, which needs to be approached from an integrated point of view in terms of electric infrastructure and electric vehicle fleet connection. In a first stage is necessary to define the high level specifications, and the optimal size for both infrastructure and electric vehicle fleet, according to the sustainable mobility expected targets of the city analyzed, e.g. mobility covered/filled areas, emission reduction, consumption reduction, cost reduction, energy \& mobility efficiencies. In order to obtain the optimum integrated solution, a global approach of the problem is essential, moving from the high level definition of electric vehicle fleet and infrastructure, to the more detailed definition of systems and subsystems derived. CITYELEC is a strategy R\&D Spanish project, analyzing, covering and solving the particular urban mobility electrification problem in some cities of Spain, starting from the high level definition of the urban mobility issues, and concluding in the detailed definition of the vehicle and infrastructure specifications. In CITYELEC project, special methodologies, global modelling and simulation tools, and advanced systems and subsystems are being under research and development, aiming for the optimum electro mobility integrated solution, including the infrastructure and the electric vehicle fleet.
\end{abstract}

Keywords: electrification, urban mobility, electric infrastructure, optimization

\section{Introduction}

CITYELEC strategic project was launched October 2009, and with a budget of $€ 19.0$ million financed partially by the Spanish Ministry of Science and Innovation, represents a milestone in the vehicle electrification $R \& D$ initiatives in Spain. The project gathers the efforts of different companies (automotive and electric), city councils of San Sebastian and Zaragoza, research centres and universities. With duration of four years the project will make possible the development of new concepts of electrical vehicles and recharge infrastructures for urban environments. CITYELEC project arises from the urban sustainable mobility concern today, showing that a large portion of the population limits their daily travel to short / medium distances of less than 80 kilometres, often within urban agglomerations.

CITYELEC project is focused on the key elements both in vehicle and infrastructure, for developing new concepts of electrified mobility in urban environment. This approach is carried out from 
high level specification (urban mobility needs) to low level product definition, with an integrated point of view (taking into account both infrastructure and vehicle), in order to create a electric vehicle fleet optimized for the mobility requirements of the cities participating in the project.

The project will last four years (2009 - 2012) and will experiment and validate EV on the road for a period of a year (2011). Its aim is to come up with conclusions and recommendations by autumn 2012. In this paper a compilation of the results obtained so far, and the global approach methodology are shown.

\section{High level CITYELEC requirements}

CITYELEC global and integrated approach aims to develop an electric vehicle fleet and the required infrastructure, starting from the urban mobility and traffic flow data from cities. Therefore, urban mobility data from two city councils participating in the project has been used for the high level requirements definition. Two type of different city topologies (compact and dispersed), and consequently different urban mobility demands are represented by the two different cities (both in the north of Spain, San Sebastian and Zaragoza). In the following lines the results of the compact city (San Sebastian) will be explained as an example, but the same methodology has been carried out with the dispersed city (Zaragoza).

\subsection{Urban transport mobility data}

San Sebastian is a compact city $(185.000$ habitants) in the north of Spain, and the urban mobility data collected in order to define the high level CITYELEC requirements, illustrate this compact topology.

The city has low to medium traffic congestion depending on the daytime, but attending to the data from the traffic authorities, and the collected data from some logged vehicles, rush hours are routine and always associated with the start and end of the working day. Even if the city is less than 60 square $\mathrm{km}$, the private vehicle is used in the $34 \%$ of the journeys. Fig. 1 shows the modal city transport distribution.

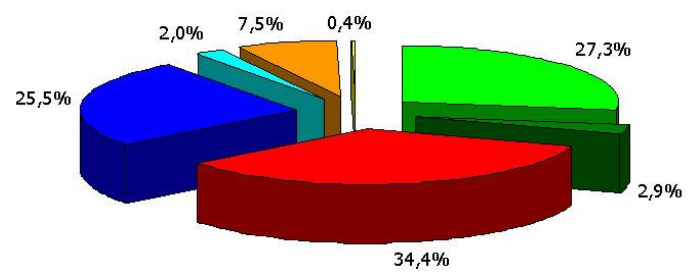

$\square$ Walking $\square$ Bicycle $\square$ Car $\quad \square$ Bus $\quad \square$ Railway $\square$ Motorcycle $\square$ Other

Figure 1: Modal city transport distribution.

Furthermore San Sebastian city is quite oriented to tourism, and this creates a heavy traffic in the central and flat zones of the city, hence these areas where some of the characteristics of the electrical vehicles are very valuable (no pollution, no noise) have been selected for the definition of the electric vehicle specifications: Fig. 2 shows the city centre map and the logging hardware installed in a electric scooter.

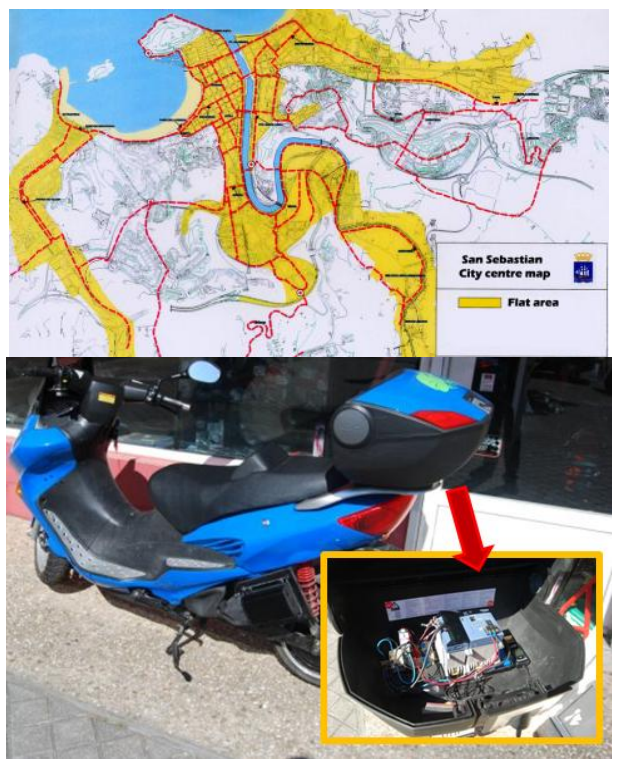

Figure 2: San Sebastian city centre map and logging hardware installed in an electric scooter.

\subsection{Electric vehicle fleet definition}

In order to define the electric vehicle fleet and his benefits, is necessary to estimate the overall $\mathrm{CO} 2$ emission reduction (taking into account the energy mix in each country), with the technical specifications and number of units of each type of vehicle on the road. The technical specifications of the vehicle and the infrastructure are quite straightforward to define with the traffic and urban 
mobility data from the city. Table 1 shows the specifications of CITYELEC vehicles.

\begin{tabular}{|c|c|c|c|}
\hline \multirow{2}{*}{$\begin{array}{c}\text { CITYELEC } \\
\text { VEHICLES }\end{array}$} & \multicolumn{3}{|c|}{ City of San Sebastian } \\
\cline { 2 - 4 } & Scooter & Citycar & $\begin{array}{c}\text { Mini } \\
\text { Bus }\end{array}$ \\
\hline $\begin{array}{c}\text { Power } \\
\text { (nominal/peak) }\end{array}$ & $5.5 \mathrm{~kW}$ & $15-30 \mathrm{~kW}$ & $\begin{array}{c}15-30 \\
\mathrm{Kw}\end{array}$ \\
\hline $\begin{array}{c}\text { Battery capacity } \\
\text { WWh }\end{array}$ & $\begin{array}{c}2.4-3.6 \\
\mathrm{kWh} \\
\mathrm{kWh}\end{array}$ & $\begin{array}{c}12-18 \\
\mathrm{kWh}\end{array}$ \\
\hline $\begin{array}{c}\text { Weight/Passeng } \\
\text { ers }\end{array}$ & $140 / 2$ & $1050 / 4$ & $\begin{array}{c}2300 / \\
15\end{array}$ \\
\hline
\end{tabular}

Table 1: CITYELEC vehicle specifications for the city of San Sebastian.

The $\mathrm{CO} 2$ emission reduction of the whole fleet is more complex to obtain, but very valuable because is a reference of the environmental benefits in terms of reduction of $\mathrm{CO} 2$ emissions. Within the CITYELEC project a $\mathrm{CO} 2$ emission calculator software has been developed, considering the energy mix of each European country, and giving the possibility of comparing an electric vehicle fleet with a conventional based on internal combustion engine. The software is based on backward modeling of the vehicle to be simulated, and different standardizes driving cycles or user defined. Therefore it permits to launch simulations of electric vehicle fleets, mix of different type of vehicles or define a particular vehicle. With these backward simulations and the data from the city, the electric vehicle fleet (number of vehicles) and the overall $\mathrm{CO} 2$ emission reduction can be estimated, and the optimal electric vehicle fleet for the selected city can be defined by iterative simulations. Fig. 3 shows the $\mathrm{CO} 2$ emission calculator software which allows estimating the optimal electric vehicle fleet.

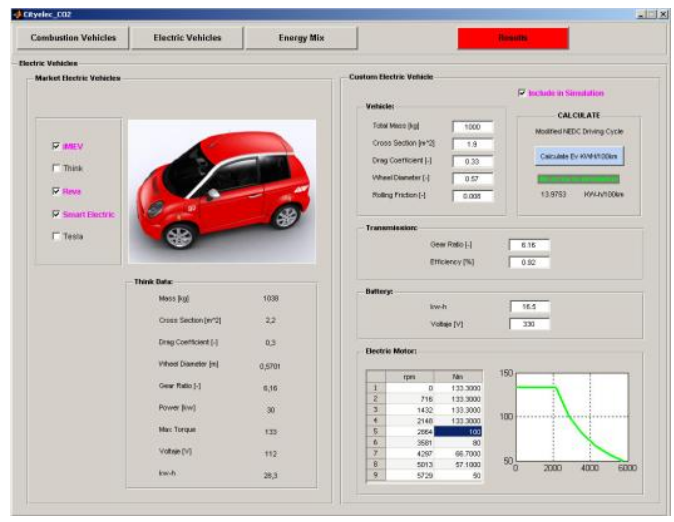

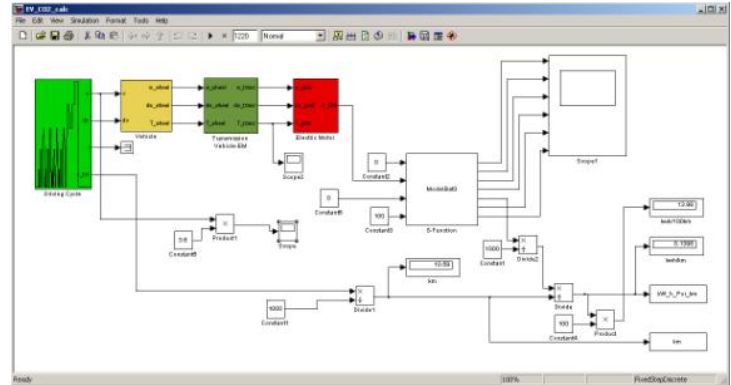

Fig. $3 \mathrm{CO} 2$ emission calculator software and backward models inside.

\section{CITYELEC vehicle electric propulsion system}

Results and methodology used to obtain the electric propulsion system specifications of the CITYELEC battery electric vehicles (city-car, citybus, and city-motorbike) will be presented. Results of the previous section were used as input and Model Based Design (MBD) as the methodology. A vehicles backward modeling and simulation tool in combination with an iterative optimization technique was developed in order to obtain the best specifications of the CITYELEC vehicle's electric propulsion subsystems. Some targets including the energy efficiency, size \& weight, reliability, cost and alignment with international R\&D automotive programs and technological road maps were considered on the search and definition of the electric propulsion functional specifications.

Different electric propulsion architectures have been evaluated such us: (1) stand alone or propulsion power centralized (electric machine with gear box \& differential) and (2) "in wheel motor" or propulsion power distributed (electric machines integrated into the wheels). As an example, table 2 shows the specifications obtained for the electric propulsion system with stand alone architecture of the CITYELEC battery electric vehicle (BEV-City-car). 


\begin{tabular}{|c|c|c|c|}
\hline \multicolumn{4}{|c|}{ Electric propulsion system specifications: BEV-City-car } \\
\hline Description & Value & Description & Value \\
\hline Continuous Power & $15 \mathrm{Kw}$ & Battery dc voltage & $260 \mathrm{v}-400 \mathrm{v}$ \\
\hline Peak Power (20s) & $30 \mathrm{Kw}$ & Battery energy & $9,6-13 \mathrm{Kwh}$ \\
\hline Drive speed: base / max & $1.000 \mathrm{rpm} / 4.000 \mathrm{rpm}$ & Battery power & $30 \mathrm{Kw}$ \\
\hline Continuous Torque & $143 \mathrm{Nm}$ & Battery weight & $<150 \mathrm{Kg}$ \\
\hline Peak Torque (20s) & $285 \mathrm{Nm}$ & Electric machine weight & $<35 \mathrm{Kg}$ \\
\hline Electric machine voltage & $150 \mathrm{v}$ & Electric machine volume & $<161$ \\
\hline Electric machine efficiency & $>90 \%(15-100 \%$ max speed) & Inverter weight & $<9 \mathrm{Kg}$ \\
\hline Inverter current (max.) & $250 \mathrm{~A}$ & Inverter volume & $<91$ \\
\hline Inverter efficiency & $>96 \%$ & Gear box weight & $<20 \mathrm{Kg}$ \\
\hline Gear box ratio & 3,864 & Gear box volume & $<31$ \\
\hline Gear box efficiency & $>90 \%$ & & \\
\hline
\end{tabular}

Table 2: Electric propulsion system specifications for CITYELEC BEV-Citycar.

Simulations results corresponding to the iteration from which these specifications were chosen are shown in figure 4. Standard USA FTP-75 driving cycle was used as input. Electric drive operation points (speed, torque) are displayed over the continuous and maximum torque vs. speed characteristic of the electric drive. Most of the operation points are placed below the continuous torque vs. speed characteristic and also concentrated in high efficiency zones. A compromise between functional and cost \& reliability targets was also considered.

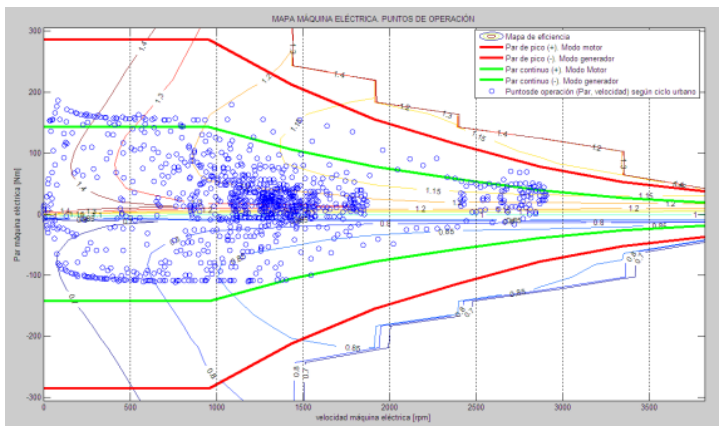

Figure 4: Simulation results for CITYELEC BEVCity-car using standard USA FTP-75.

Some R\&D actions have been initiated with some Spanish industrial partners with the aim to fulfill the specifications obtained for the different electric propulsion subsystems. Figure 5 shows some results related with the conceptual design of a Permanent Magnet Synchronous Machine (PMSM) for the BEV-city-car with stand alone configuration. In the figure 6 , the conceptual design related with the integration of a ten kilowatts axial flux PMSM into the wheel, is presented.

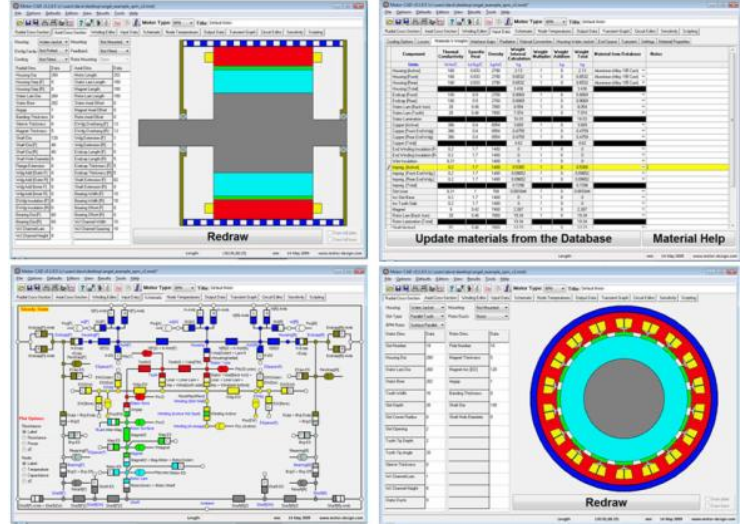

Figure 5: Conceptual design of a radial flux PMSM for CITYELEC-BEV city-car with stand alone configuration.
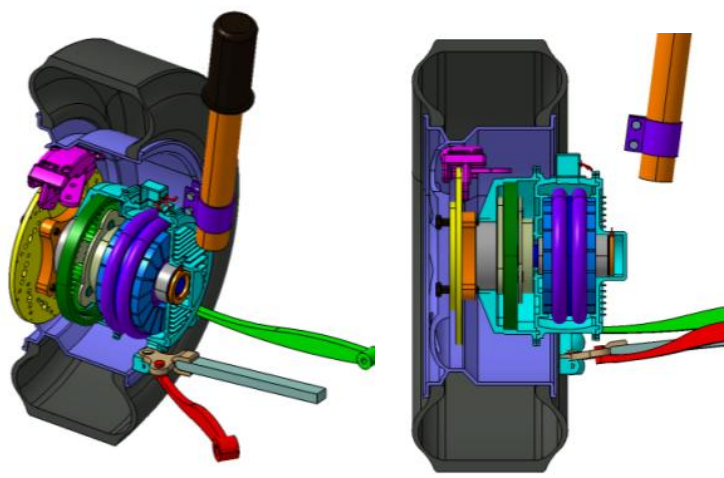

Figure 6: In wheel integration of a $10 \mathrm{Kw}$. axial flux PMSM for CITYELEC-BEV city-car.

\section{CITYELEC infrastructure}

electric

In this section, the results and methodology used to size and define the characteristics of the electric infrastructure needed in the cities, under the CITYELEC approach, will be presented.

Main objective resides on the design and the sizing of the electric infrastructure subsystems needed to support the CITYELEC vehicles' fleet. Electric infrastructure will be designed and introduced in an environment friendly way, according with the characteristics of the CITYELEC model approach. Some infrastructure subsystems are being developed, with next operational objectives:

Intermediate Energy Store System (IESS): Intermediate energy store systems between the primary energy source and the electric vehicles' fleet. They will have a great energy \& power capacity to guarantee the vehicles' fleet energy 
supply. This accumulated energy will help on the balancing of the power grid. Electric vehicles could be recharged even during peak hours without increasing the grid power consumption. At off-peak hours the IESS could be recharged, helping on the power plants and electrical system balancing. Thus, they could also attenuate the variability of the renewable power plants.

BEV Recharge Points with Bidirectional Communication System: Aspects such us: power consumption measurements, user credentials, rate-settings, recharge management, security, etc., should be implemented, controlled and supervised in all the recharge points. A bidirectional communication system between the recharge points and the energy distribution management system is mandatory.

Integration of renewable energy in the cities: The BEV is as clean as the primary energy used for his recharge. Our cities have some particular restrictions for the introduction of micro grids based on renewable energy. Evaluation studies of the most appropriated technologies for his introduction on the cities are being done in order to develop some prototypes.

\section{Conclusion}

An example of global solution approach for the electrification of urban mobility in Spain has been presented in this paper, showing that the optimal sizing of both electric vehicleinfrastructures requires a comprehensive approach, taking into consideration the city topology and urban mobility issues, that will be the electric vehicles and infrastructure defining input data.

\section{Acknowledgments}

This work comes under the framework of the CITYELEC research project PSE 370000-20094. This research project is being granted by the Spanish Ministry of Science and Innovation (National Applied Research Program).

\section{References}

[1] Perujo, A. and Ciuffo, B. 'Potential Impact of Electric Vehicles on the Electric Supply System; A case study for the Province of Milan, Italy', Office for Official Publications of the European Communities, Luxembourg, September 2009
[2] European Automotive Manufacturers 'The Electrification of the Vehicle and the Urban Transport System', Recommendations on key $\mathrm{R} \& \mathrm{D}$ by the European Automotive Manufacturers.

[3] Schrank, D. and Lomax, T. '2009 URBAN MOBILITY REPORT' Texas Transportation Institute The Texas A\&M University System.

[4] Chau K.T. et. Al. "Overview of power electronic drives for electric vehicles". HAIT Journal of Science and Engineering B. Vol.2 p. 737-761.

[5] Ehsani M. et. Al. "Characterization of electric motor drives for traction applications". Industrial Electronics Society, IECON'03 The 29th Annual Conference of the IEEE. Vol.1 p.891-896.

[6] Guzzella L. et. Al. "CAE Tools for QuasiStatic Modeling and Optimization of Hybrid Powertrains". IEEE Transactions on Vehicular Technology. Vol.48 p. 1762-1769.

[7] Technology Road Map. Electric and Plug-In Hybrid Electric Vehicles. IEA, International Energy Agency IEA, 2009. 52p.

[8] Dr. Duane Hanselman. Brushless Permanent Magnet Motor Design. 2nd edition. Electrical and Computer Engineering University of Maine Orono, USA. Magna Physics Publishing, 2006. 392p. ISBN: 1-881855-155

\section{Authors}

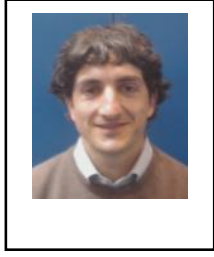

Mr. Iñaki Iglesias Aguinaga

Tel: +3494600 2266 Fax: +3494 6002299

Email: inaki.iglesias@tecnalia.com Material Engineer, 2001 TECNUN, University of Navarra, Spain. Actually working as researcher in Tecnalia Research \& Innovation involved in the vehicle dynamics and alternative powertrain area, with focus on developments on vehicle dynamics and design with DiL and HiL.

\section{Mr. Juan José Valera Garcia}

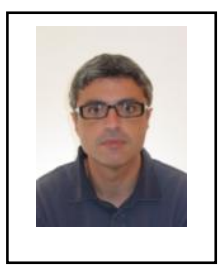

Tel: +3494 6002266 Fax: +3494 6002299

Email: juanjose.valera@tecnalia.com Industrial Electronic Technical Engineer, 1992, University of Mondragon, Spain. Electronic and Automatic Control Sup. Engineer (2008), University of Bilbao, Spain. Actually working as researcher in Tecnalia Research \& Innovation Alternative Powertrain area. 
